\title{
Apoptosis-independent cleavage of RhoGDI $\beta$ at Asp19 during PMA-stimulated differentiation of THP-1 cells to macrophages
}

\author{
TAKAHIDE OTA $^{1}$, YONG-SHENG JIANG ${ }^{2}$, MAMORU FUJIWARA $^{3}$ and MASAAKI TATSUKA ${ }^{3}$ \\ ${ }^{1}$ Division of Tumor Biology, Department of Life Science, Medical Research Institute, Kanazawa Medical University, \\ Uchinada, Ishikawa 920-02, Japan; ${ }^{2}$ Department of Oncology, Tongji Hospital of Tongji Medical College, \\ Huazhong University of Science and Technology, Wuhan, Hubei 430030, P.R. China; ${ }^{3}$ Department of Life Sciences, \\ Life and Environmental Sciences, Prefectural University of Hiroshima, Shoubara, Hiroshima 727-0023, Japan
}

Received January 13, 2016; Accepted January 5, 2017

DOI: $10.3892 / \mathrm{mmr} .2017 .6199$

\begin{abstract}
Rho GDP-dissociation inhibitor $\beta$ (RhoGDI $\beta$ ), a regulator of the Rho family of proteins, is expressed abundantly in the hematopoietic cell lineage. During apoptosis of hematopoietic cells, RhoGDI $\beta$ is cleaved by caspase-3 at Asp19 and this cleaved form $(\Delta 19-$ RhoGDI $\beta)$ has been implicated in the apoptotic pathway. To clarify the role of RhoGDI $\beta$ in hematopoietic cells, the present study performed immunoblotting and immunofluorescence staining to examine the expression of RhoGDI $\beta$ and $\Delta 19$-RhoGDI $\beta$ during phorbol 12-myristate 13-acetate (PMA)-stimulated differentiation of human THP-1 monocytic cells to macrophages. During differentiation of the THP-1 cells to macrophages, the expression of RhoGDI $\beta$ remained stable; however, the expression of $\Delta 19$-RhoGDI $\beta$ increased, particularly in well-spreading, non-apoptotic cells, which differentiated into macrophages. These results suggested that $\Delta 19$-RhoGDI $\beta$ has an apoptosis-independent role in the PMA-induced differentiation of THP-1 cells to macrophages.
\end{abstract}

\section{Introduction}

Rho GDP-dissociation inhibitors (RhoGDIs) are regulators of the Rho family of proteins and function as molecular switches in numerous cellular processes, including actin cytoskeletal organization, microtubule dynamics, vesicle trafficking,

Correspondence to: Dr Takahide Ota, Division of Tumor Biology, Department of Life Science, Medical Research Institute, Kanazawa Medical University, 1-1 Daigaku, Uchinada, Ishikawa 920-02, Japan E-mail: takahide@kanazawa-med.ac.jp

Abbreviations: BSA, bovine serum albumin; GAP, GTPaseactivating protein; GEF, guanine nucleotide exchange factor; PBS, phosphate-buffered saline; PMA, phorbol 12-myristate 13-acetate; RhoGDI, Rho GDP-dissociation inhibitor; SDS, sodium dodecyl sulphate

Key words: caspase-3, differentiation, Rho GDP-dissociation inhibitor, THP-1, macrophages, phorbol 12-myristate 13-acetate cell polarity and cell cycle progression (1). A total of three RhoGDIs (RhoGDI $\alpha /$ RhoGDI1, RhoGDI/RhoGDI2/LyGDI/ D4GDI and RhoGDI $\gamma /$ RhoGDI3) have been identified in mammals (2). RhoGDI $\beta$ is expressed abundantly in hematopoietic cells $(3,4)$ and is also expressed in non-hematopoietic cells, including keratinocytes, fibroblasts and amnion cells (5), non-hematopoietic tumors (6-8) and various cancer cells (9).

During apoptosis of hematopoietic cells, RhoGDI $\beta$ is cleaved by caspase-3 at Asp19 (10-16), and the cleaved form, $\Delta 19$-RhoGDI $\beta$, translocates to the nucleus $(12,15-17)$, suggesting the pro-apoptotic role of $\Delta 19$-RhoGDI $\beta$. Therefore, the cleavage of RhoGDI $\beta$ by caspase-3 has been implicated in the apoptotic pathway, however, the precise role of $\Delta 19-$ RhoGDI $\beta$ remains to be elucidated. Caspase-3 is known to have apoptosis-independent roles in the differentiation and proliferation of various cell types, including hematopoietic cells (18). In the differentiation of monocytes into macrophages, caspase- 3 and caspase- 9 are activated, and the inhibition of these caspases prevents this differentiation (19). Furthermore, phorbol 12-myristate 13-acetate (PMA)-stimulated THP-1 cells express increased levels of caspase-3 (20). However, whether RhoGDI $\beta$ is cleaved at Asp19 during the differentiation of these cells remains to be elucidated.

In the present study, to clarify the role of $\Delta 19$-RhoGDI $\beta$ in the differentiation of macrophages, the expression of RhoGDI $\beta$ and $\Delta 19$-RhoGDI $\beta$ were examined during PMA-stimulated differentiation of human THP-1 monocytic cells to macrophages. The results confirmed that RhoGDI $\beta$ was cleaved at Asp19 and it was shown that this cleaved form was expressed in non-apoptotic cells. These results suggested the apoptosis-independent role of $\Delta 19-\mathrm{RhoGDI} \beta$ during the differentiation of THP-1 cells to macrophages.

\section{Materials and methods}

Cell culture and induction of differentiation. The THP-1 human myelomonocytic cell line was provided by Dr Masaharu Wano, Department of Hematology and Immunology, Kanazawa Medical University (Uchinada, Japan). The cells were cultured in RPMI 1640 medium containing $2 \mathrm{mM} \mathrm{L}$-glutamine (Sigma-Aldrich; Merck Millipore, Darmstadt, Germany) supplemented with $10 \%$ fetal bovine serum (Invitrogen; 
Thermo Fisher Scientific, Inc., Waltham, MA, USA), and were maintained at $37^{\circ} \mathrm{C}$ in a humidified atmosphere of $5 \% \mathrm{CO}_{2}$ in air. The differentiation of THP-1 cells into macrophages was achieved using the method described by Daigneault et al (21). Briefly, THP-1 cells $\left(\sim 1.5 \times 10^{5} / \mathrm{ml}\right)$ were cultured with $200 \mathrm{nM}$ PMA (Sigma-Aldrich; Merck Millipore) for 3 days at $37^{\circ} \mathrm{C}$, the PMA-containing media was removed and the cells were incubated for a further 5 days. For counting the total cell number, the culture medium containing floating cells was removed and reserved, and the attached cells were detached using $0.25 \%$ Trypsin $/ 0.01 \%$ EDTA in phosphate-buffered saline (PBS), following which they were suspended with the previously reserved medium containing the floating cells. The cell numbers were then counted using a hemocytometer. The relative numbers of flattened cells on the dish were observed under a phase-contrast microscope. The proportion of flattened cells was estimated as it was difficult to distinguish between flattened and unflattened cells precisely.

Antibodies. Anti-RhoGDI $\beta$ antibody (cat. no. sc-6047) raised against amino acid residues 175-194 was purchased from Santa Cruz Biotechnology, Inc. (Santa Cruz, CA, USA). This antibody recognizes full-length and $\Delta 19$-RhoGDI $\beta$. Anti- $\Delta 19$-RhoGDI $\beta$ antibody (clone 97A1015; cat. no. 14-6628-81) raised against the caspase- 3 cleavage site of human RhoGDI $\beta$ was purchased from eBioscience, Inc. (San Diego, CA, USA). Anti-a-tubulin antibody (clone B-5-1-2; cat. no. T6074) was purchased from Sigma-Aldrich; Merck Millipore. Peroxidase-conjugated anti-mouse (cat. no; K4001) and anti-rabbit IgG antibodies (cat. no. K4002) were purchased from DakoCytomation (Glostrup, Denmark). Peroxidase-conjugated anti-goat IgG antibody (cat. no; 414351) was purchased from the Nichirei Corporation (Tokyo, Japan). Alexa Fluor 594-conjugated goat anti-mouse IgG (H+L; cat. no. A-11032) was purchased from Invitrogen; Thermo Fisher Scientific, Inc.

Immunoblotting. The cells not exposed to PMA (untreated cells) were found not to attach to the culture dish, whereas $>95 \%$ of the PMA-stimulated cells attached. When the cell lysates of the PMA-stimulated cells were prepared, floating cells and cell debris were removed to avoid contamination by the dead cells. The cells were lysed using Laemmli buffer containing 4\% sodium dodecyl sulfate (SDS), $20 \%$ glycerol, 10\% 2-mercaptoethanol, $0.004 \%$ bromophenol blue and $0.0125 \mathrm{M}$ Tris- $\mathrm{HCl}(\mathrm{pH} 6.8)$, and the protein concentrations of the lysate were measured using a Bradford Ultra kit (Novexin, Ltd., Cambridge, UK). The proteins $(10 \mu \mathrm{g})$ were resolved by SDS-polyacrylamide gel electrophoresis and transferred onto Immobilon-P membranes (EMD Millipore, Billerica, MA, USA). The membranes were then probed with a primary antibody (sc-6047, 1:10,000 dilution; clone 97A1015, 1:10,000 dilution; clone B-5-1-2, 1:100,000 dilution) overnight at $4^{\circ} \mathrm{C}$, followed by incubation with a peroxidase-conjugated secondary antibody (1:500 dilution) for $90 \mathrm{~min}$ at room temperature. The immunoreactive proteins were visualized using ECL Prime reagents (GE Healthcare Life Sciences, Ltd., Little Chalfont, UK). The same quantity of protein was applied in all immunoblot experiments.

Annexin $V$ and immunofluorescence staining. The cells were grown in 35-mm culture dishes. To remove dead and apoptotic cells, the dishes were washed twice with PBS at $4^{\circ} \mathrm{C}$. The cells were stained with the Annexin V-FITC Apoptosis Detection kit I (BD Biosciences, San Jose, CA, USA) according to the manufacturer's protocol, and fixed with freshly prepared $3.7 \%$ paraformaldehyde in Annexin $\mathrm{V}$ binding buffer containing $140 \mathrm{mM} \mathrm{NaCl}, 2.5 \mathrm{mM} \mathrm{CaCl}_{2}$ and $10 \mathrm{mM} \operatorname{HEPES}(\mathrm{pH} 7.5$ ) for $30 \mathrm{~min}$ at room temperature. The cells were then permeabilized with $0.5 \%$ Triton X-100 for $5 \mathrm{~min}$ at room temperature. Following washing with PBS, the cells were incubated with $0.5 \%$ bovine serum albumin (BSA) in PBS for $60 \mathrm{~min}$ at room temperature, and then incubated overnight at $4^{\circ} \mathrm{C}$ with anti- $\Delta 19$-RhoGDI $\beta$ antibody (clone 97A1015) diluted 1:400 in PBS containing $0.5 \%$ BSA. Following three washes with PBS, the cells were incubated for $60 \mathrm{~min}$ at room temperature with a secondary antibody (A-11032), diluted 1:400 in PBS containing $0.5 \% \mathrm{BSA}$ and $0.1 \mu \mathrm{g} / \mathrm{ml} \mathrm{4}$,6-diamidino-2-phenylindole. Following three washes with PBS, the cells were mounted with ProLong Gold (Invitrogen; Thermo Fisher Scientific, Inc.). Images were captured using an Axiovert 200 inverted fluorescence microscope (Plan Neofluar 40X/0.75 NA objective lens) with AxioVision 4.4 software (Carl Zeiss AG, Jena, Germany). Images of the green, red and blue channels were captured using a 38HE bandpass filter (excitation, 450-490 nm; emission, 500-550 nm), a 43HE bandpass filter (excitation, 537-563 nm; emission 570-640 nm) and a 49 bandpass filter (excitation, G $365 \mathrm{~nm}$; Emission, 420-470 nm), respectively.

\section{Results}

Induction of THP-1 cell differentiation into macrophages. The THP-1 cells were cultured with 200 nM PMA for 3 days, following which the PMA-containing media was removed and the cells were then incubated for a further 5 days (Fig. 1). Cell proliferation was suppressed and the proportion of attached cells increased considerably within 2 days. In addition $>90 \%$ of the attached cells were flattened 2 days following treatment with PMA. The differentiation of THP-1 cells to macrophages is known to be accompanied by a loss of proliferation (22); furthermore, cell adhesion and spreading are used as functional indicators of macrophage differentiation $(21,23)$. The results obtained indicated that the majority of THP-1 cells were induced to differentiate into macrophages.

The cells cultured for 5 days following PMA removal showed a higher degree of flattened morphology, compared with the cells cultured for 3 days with PMA (Fig. 1, top panel). It has been reported that cultures rested for 5 days in PMA-free media adopt a phenotype, which more closely resembles human monocyte-derived macrophages, compared with differentiated THP-1 cells cultured for only 3 days with PMA (21). The flattened morphology observed in the present study of the cells cultured 5 days following PMA removal is likely to reflect the increased degree of differentiation of these cells.

Expression of $419-R h o G D I$ in THP-1 cells differentiated into macrophages. The present study used immunoblotting to examine the expression of RhoGDI $\beta$ and its cleaved form at Asp19 during the PMA-stimulated differentiation of THP-1 cells to macrophages. Following treatment with PMA, the expression of full-length RhoGDI $\beta$ remained unchanged, 


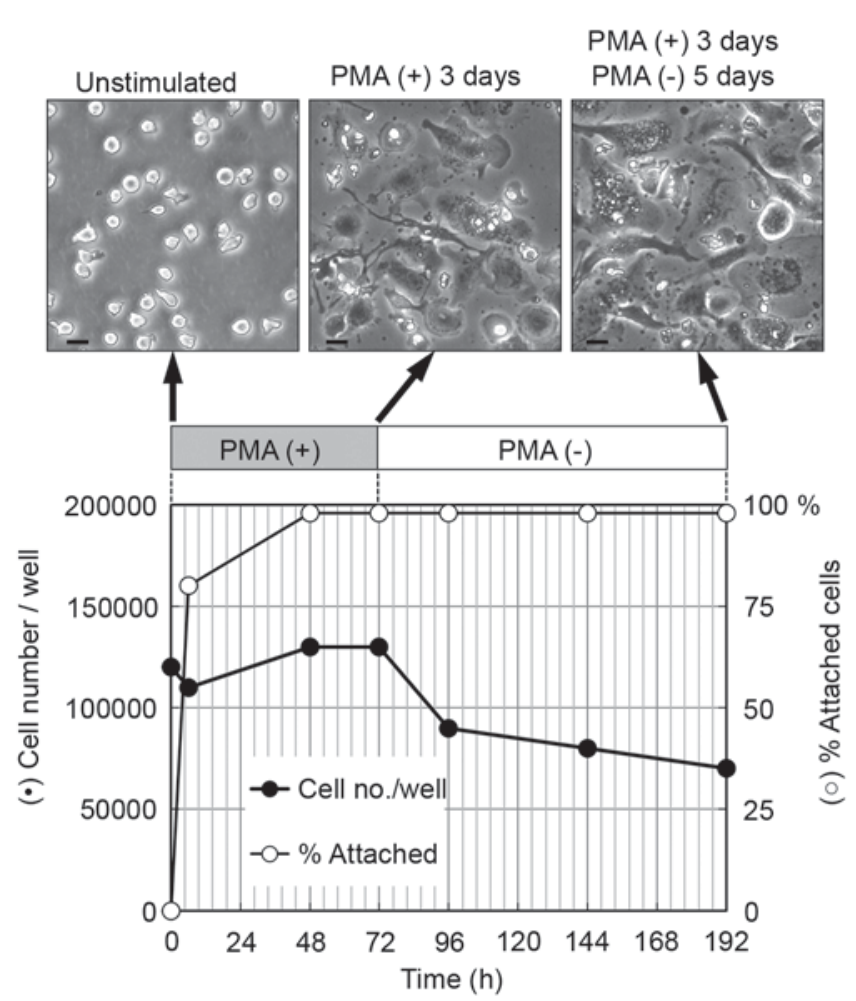

Figure 1. Induction of THP-1 cell differentiation into macrophages by PMA. Cells were seeded with $200 \mathrm{nM}$ PMA in a 24-well plate and cultured for 3 days, following which the cells were cultured without PMA for a further 5 days. Total cell number and the proportion of attached cells were measured. Scale bar $=20 \mu \mathrm{m}$. Similar results were obtained for three independent experiments. Representative results are shown. PMA, phorbol 12-myristate 13 -acetate.

whereas the expression of $\Delta 19$-RhoGDI $\beta$ increased and was correlated with the differentiation of the cells into macrophages, although only a small fraction of full-length RhoGDI $\beta$ was cleaved at Asp19 (Fig. 2, top panel). The increased expression of $\Delta 19$-RhoGDI $\beta$ was confirmed using anti- $\Delta 19-$ RhoGDI $\beta$ antibody (97A1015; Fig. 2, middle panel). The expression level of $\Delta 19$-RhoGDI $\beta$ was greater in cells cultured 5 days following PMA removal, compared with cells cultured for 3 days only with PMA. Therefore, the expression level of $\Delta 19$-RhoGDI $\beta$ appeared to correlate with the degree of differentiation into macrophages. To confirm the expression of $\Delta 19$-RhoGDI $\beta$ in macrophage-differentiated THP-1 cells, the differentiated cells were stained with anti- $\Delta 19-\mathrm{RhoGDI} \beta$ antibody. $\Delta 19$-RhoGDI $\beta$ was detected in the macrophage-differentiated THP-1 cells (Fig. 3A).

During the differentiation of THP-1 cells to macrophages, the total cell number gradually decreased (Fig. 1). As low levels of apoptotic cell death are reported to occur in macrophage-differentiated THP-1 cells in the absence of apoptotic stimuli (24), the decrease in cell number observed in the present study was likely due to apoptotic cell death. To exclude the possibility that the detected levels of $\Delta 19-\operatorname{RhoGDI} \beta$ in the differentiated cells were derived from apoptotic cells, the differentiated cells attached to the culture dish were stained with Annexin V and anti- $\Delta 19-$ RhoGDI $\beta$ antibody (Fig. 3B). The unattached dead and apoptotic cells were almost completely removed by washing with PBS prior to staining. The proportion of cells stained with Annexin V was $<1 \%$.

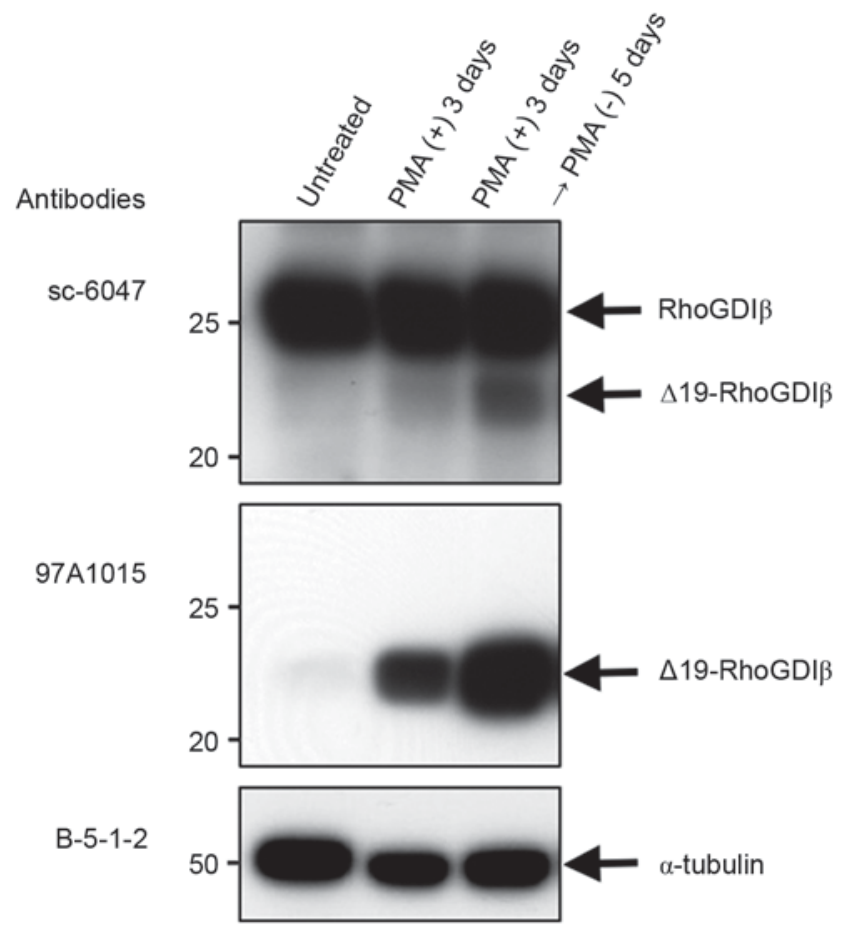

Figure 2. Expression of RhoGDI $\beta$ during the differentiation of THP-1 cells to macrophages. For the sampling of differentiation-induced cells, unattached dead and apoptotic cells were removed. The cell lysate was immunoblotted with an antibody (sc-6047), which recognizes full-length RhoGDI $\beta$ and $\Delta 19$-RhoGDI $\beta$, and an antibody (97A1015), which specifically recognizes $\Delta 19$-RhoGDI $\beta$. $\alpha$-tubulin was stained with B-5-1-2 antibody as a loading control. Similar results were obtained for three independent experiments. Representative results are shown. RhoGDI $\beta$, Rho GDP-dissociation inhibitor $\beta$.

The observed Annexin V-positive cells are shown in Fig. 3B and C. $\Delta 19$-RhoGDI $\beta$ was detected in the apoptotic cells and macrophage-differentiated THP-1 cells, and the majority of the expression of $\Delta 19$-RhoGDI $\beta$ was observed in the non-apoptotic cells, which differentiated into macrophages.

\section{Discussion}

During the apoptosis of hematopoietic cells, RhoGDI $\beta$ is cleaved by caspase-3 at Asp19 (10-16), and the cleaved form, $\Delta 19$-RhoGDI $\beta$, has been suggested to have a pro-apoptotic function in K562 leukemia cells (17). In the present study, it was shown that, during the PMA-stimulated differentiation of THP-1 cells to macrophages, RhoGDI $\beta$ was also cleaved at Asp19 in non-apoptotic differentiating cells. Although it is unknown whether $\Delta 19$-RhoGDI $\beta$ is involved in the differentiation process or the differentiation phenotype, these results suggested that $\Delta 19-\mathrm{RhoGDI} \beta$ is involved in cellular processes other than apoptosis, at least in THP-1 cells. $\Delta 19$-RhoGDI $\beta$ may have different roles depending on the cellular context.

RhoGDI $\beta$ is implicated in cancer progression; however, the correlation between malignancy and the expression level of RhoGDI $\beta$ is contradictory (25). It has been previously reported that RhoGDI $\beta$ may have a positive $(7,26,27)$ and negative $(28)$ role in cancer progression. Caspase-3 is considered to be involved in cancer susceptibility in squamous-cell carcinomas of the head and neck (29), endometrial cancer (30) and lung 
A

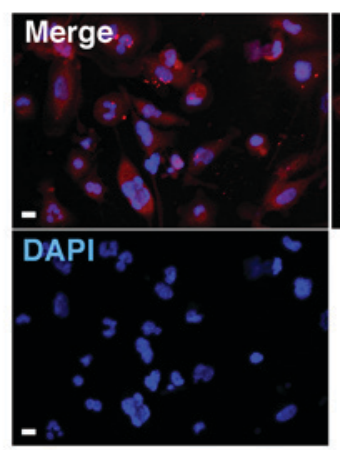

C
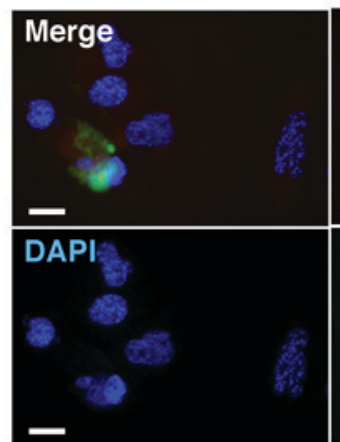
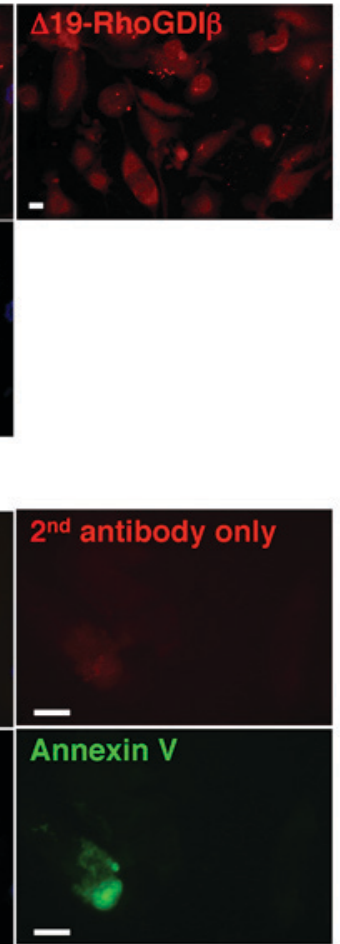

B

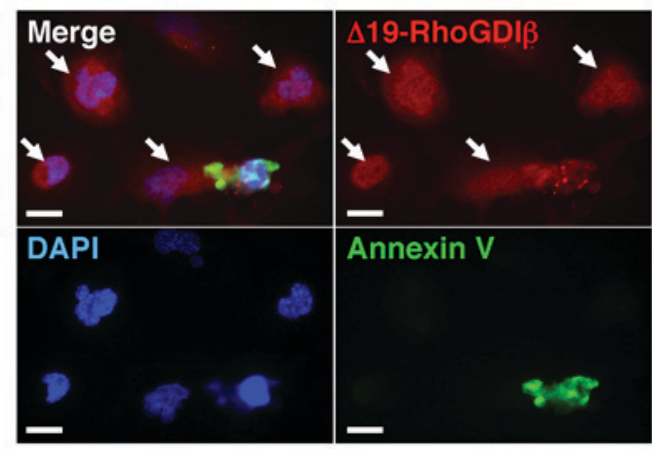

D

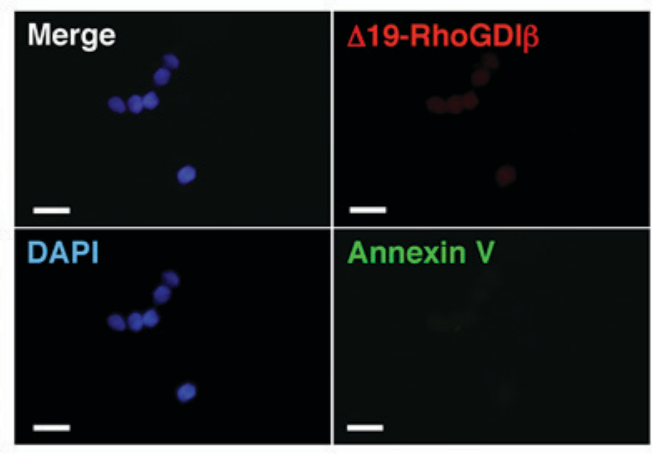

Figure 3. Expression of $\Delta 19$-RhoGDI $\beta$ in non-apoptotic cells during the differentiation of THP-1 cells to macrophages. For immunostaining of differentiation-induced cells unattached dead and apoptotic cells were removed. The cells were cultured with $200 \mathrm{nM}$ PMA for 3 days, and then cultured without PMA for a further 5 days. (A) Differentiated cells were stained with anti- $\Delta 19$-RhoGDI $\beta$ antibody (red). (B) Differentiated cells were stained with Annexin V (green) and anti- $\Delta 19$-RhoGDI $\beta$ antibody (red). Arrows indicate $\Delta 19$-RhoGDI $\beta$-positive non-apoptotic cells. (C) Differentiated cells stained with Annexin V (green) and only with secondary antibody (red). (D) Unstimulated cells were stained with Annexin V (green) and anti- $\Delta 19$-RhoGDI $\beta$ antibody (red). Scale bar=20 $\mu$ m. Similar results were obtained for three independent experiments. Representative results are shown. RhoGDI $\beta$, Rho GDP-dissociation inhibitor $\beta$; PMA, PMA, phorbol 12-myristate 13-acetate; DAPI, 4',6-diamidino-2-phenylindole.

cancer (31), therefore, RhoGDI $\beta$ may be cleaved by casapse- 3 in various cancer cells. The inconsistent correlation of RhoGDI $\beta$ with malignancy may be a reflection of the different functions of $\Delta 19$-RhoGDI $\beta$ in different types of cancer.

The function of $\Delta 19-\mathrm{RhoGDI} \beta$ remains to be elucidated, and cleavage of RhoGDI $\beta$ at Asp19 is unlikely to result in the inactivation of RhoGDI $\beta$. $\Delta 22$-RhoGDI $\alpha(32,33)$ and $\Delta 20$-RhoGDI $\alpha$ (34) have been reported to be almost fully functional for extracting GTPases from the membrane, although the inhibition of GTPase activity by $\Delta 20$-RhoGDI $\alpha$ is less effective, compared with that by wild-type RhoGDI $\alpha$ (34). The amino acid sequence and protein structure are similar between RhoGDI $\alpha$ and RhoGDI $\beta$; therefore, $\Delta 19-$ RhoGDI $\beta$ is expected to retain certain regulatory functions of full-length RhoGDI $\beta$. Our previous study reported that RhoGDIs may act as a positive and negative regulator of Rho GTPases, depending on their expression level, and their affinity for guanine nucleotide exchange factors (GEFs) and GTPase-activating proteins (GAPs) (35). The cleavage of RhoGDI $\beta$ at Asp19 may modify the regulatory function of RhoGDI $\beta$ towards Rho GTPases by altering the affinity of RhoGDI $\beta$ to GEFs and GAPs.

In conclusion, the present study showed that $\Delta 19-\mathrm{RhoGDI} \beta$ was expressed in non-apoptotic, differentiating cells, thereby suggesting an apoptosis-independent role for this protein. The identification of this novel function of $\Delta 19-$ RhoGDI $\beta$ may assist in further elucidating the regulatory system of Rho GTPases, which is involved in the regulation of numerous cellular processes.

\section{Acknowledgements}

Mr. Mamoru Fujiwara and Dr Masaaki Tatsuka (Department of Life Sciences, Life and Environmental Sciences, Prefectural University of Hiroshima, Shoubara, Japan) were supported by grants from the Prefectural University of Hiroshima Important Research Project; Interdisciplinary/Priority Research (grant nos. (S) H-26 and (S) H-27).

\section{References}

1. Etienne-Manneville S and Hall A: Rho GTPases in cell biology. Nature 420: 629-635, 2002

2. Dovas A and Couchman JR: RhoGDI: Multiple functions in the regulation of Rho family GTPase activities. Biochem J 390: 1-9, 2005.

3. Lelias JM, Adra CN, Wulf GM, Guillemot JC, Khagad M, Caput D and Lim B: cDNA cloning of a human mRNA preferentially expressed in hematopoietic cells and with homology to a GDP-dissociation inhibitor for the rho GTP- binding proteins. Proc Natl Acad Sci USA 90: 1479-1483, 1993.

4. Scherle P, Behrens T and Staudt LM: Ly-GDI, a GDP-dissociation inhibitor of the RhoA GTP-binding protein, is expressed preferentially in lymphocytes. Proc Natl Acad Sci USA 90: 7568-7572, 1993.

5. Leffers H, Nielsen MS, Andersen AH, Honoré B, Madsen P, Vandekerckhove $J$ and Celis JE: Identification of two human Rho GDP dissociation inhibitor proteins whose overexpression leads to disruption of the actin cytoskeleton. Exp Cell Res 209: 165-174, 1993.

6. Gildea JJ, Seraj MJ, Oxford G, Harding MA, Hampton GM, Moskaluk CA, Frierson HF, Conaway MR and Theodorescu D: RhoGDI2 is an invasion and metastasis suppressor gene in human cancer. Cancer Res 62: 6418-6423, 2002. 
7. Ota T, Maeda M, Suto S and Tatsuka M: LyGDI functions in cancer metastasis by anchoring Rho proteins to the cell membrane. Mol Carcinog 39: 206-220, 2004.

8. Seraj MJ, Harding MA, Gildea JJ, Welch DR and Theodorescu D: The relationship of BRMS1 and RhoGDI2 gene expression to metastatic potential in lineage related human bladder cancer cell lines. Clin Exp Metastasis 18: 519-525, 2000.

9. Harding MA and Theodorescu D: RhoGDI signaling provides targets for cancer therapy. Eur J Cancer 46: 1525-1559, 2010.

10. Essmann F, Wieder T, Otto A, Müller EC, Dörken B and Daniel PT: GDP dissociation inhibitor D4-GDI (Rho-GDI 2), but not the homologous rho-GDI 1, is cleaved by caspase-3 during drug-induced apoptosis. Biochem J 346 Pt 3: 777-783, 2000.

11. Kettritz R, Xu YX, Faass B, Klein JB, Müller EC, Otto A, Busjahn A, Luft FC and Haller H: TNF-alpha-mediated neutrophil apoptosis involves Ly-GDI, a Rho GTPase regulator. J Leukoc Biol 68: 277-283, 2000.

12. Krieser RJ and Eastman A: Cleavage and nuclear translocation of the caspase 3 substrate Rho GDP-dissociation inhibitor, D4-GDI, during apoptosis. Cell Death Differ 6: 412-419, 1999.

13. Na S, Chuang TH, Cunningham A, Turi TG, Hanke JH, Bokoch GM and Danley DE: D4-GDI, a substrate of CPP32, is proteolyzed during Fas-induced apoptosis. J Biol Chem 271: 11209-11213, 1996.

14. Rickers A, Brockstedt E, Mapara MY, Otto A, Dörken B and Bommert K: Inhibition of CPP32 blocks surface IgM-mediated apoptosis and D4-GDI cleavage in human BL60 Burkit lymphoma cells. Eur J Immunol 28: 296-304, 1998.

15. Thiede B, Siejak F, Dimmler C and Rudel T: Prediction of translocation and cleavage of heterogeneous ribonuclear proteins and Rho guanine nucleotide dissociation inhibitor 2 during apoptosis by subcellular proteome analysis. Proteomics 2: 996-1006, 2002

16. Zhou X, Suto S, Ota T and Tatsuka M: Nuclear Translocation of Cleaved LyGDI dissociated from Rho and Rac during Trp53-dependent ionizing radiation-induced apoptosis of thymus cells in vitro. Radiat Res 162: 287-295, 2004.

17. Choi MR, Groot M and Drexler HC: Functional implications of caspase-mediated RhoGDI2 processing during apoptosis of HL60 and K562 leukemia cells. Apoptosis 12: 2025-2035, 2007.

18. Shalini S, Dorstyn L, Dawar S and Kumar S: Old, new and emerging functions of caspases. Cell Death Differ 22: 526-539, 2015.

19. Sordet O, Rébè C, Plenchette S, Zermati Y, Hermine O, Vainchenker W, Garrido C, Solary E and Dubrez-Daloz L: Specific involvement of caspases in the differentiation of monocytes into macrophages. Blood 100: 4446-4453, 2002.

20. Liao HS, Matsumoto A, Itakura H, Pittman T, Kodama $\mathrm{T}$ and Geng YJ: De novo expression of the class-A macrophage scavenger receptor conferring resistance to apoptosis in differentiated human THP-1 monocytic cells. Cell Death Differ 6: 245-255, 1999.

21. Daigneault M, Preston JA, Marriott HM, Whyte MK and Dockrell DH: The Identification of Markers of Macrophage differentiation in PMA-stimulated THP-1 cells and monocyte-derived macrophages. PLoS One 5: e8668, 2010.
22. Schwende H, Fitzke E, Ambs P and Dieter P: Differences in the state of differentiation of THP-1 cells induced by phorbol ester and 1,25-dihydroxyvitamin D3. J Leukoc Biol 59: 555-561, 1996.

23. Auwerx J: The human leukemia cell line, THP-1: A multifacetted model for the study of monocyte-macrophage differentiation. Experientia 47: 22-31, 1991

24. Vicca S, Hennequin C, Nguyen-Khoa T, Massy ZA, Descamps-Latscha B, Drueke TB and Lacour B: Caspase-dependent apoptosis in THP-1 cells exposed to oxidized low-density lipoproteins. Biochem Biophys Res Commun 273: 948-954, 2000

25. Griner EM and Theodorescu D: The faces and friends of RhoGDI2. Cancer Metastasis Rev 31: 519-528, 2012.

26. Jiang YS, Maeda M, Okamoto M, Fujii M, Fukutomi R, Hori M, Tatsuka M and Ota T: Centrosomal localization of RhoGDI $\beta$ and its relevance to mitotic processes in cancer cells. Int J Oncol 42: 460-468, 2013

27. Ota T, Maeda M, Murakami M, Takegami T, Suto S and Tatsuka M: Activation of Racl by Rho-guanine nucleotide dissociation inhibitor-beta with defective isoprenyl-binding pocket. Cell Biol Int 31: 92-96, 2007.

28. Ota T, Maeda M, Sakita-Suto S, Zhou X, Murakami M, Takegami T and Tatsuka M: RhoGDIbeta lacking the N-terminal regulatory domain suppresses metastasis by promoting anoikis in v-src-transformed cells. Clin Exp Metastasis 23: 323-334, 2006.

29. Chen K, Zhao H, Hu Z, Wang LE, Zhang W, Sturgis EM and Wei Q: CASP3 polymorphisms and risk of squamous cell carcinoma of the head and neck. Clin Cancer Res 14: 6343-6349, 2008

30. Xu HL, Xu WH, Cai Q, Feng M, Long J, Zheng W, Xiang YB and Shu XO: Polymorphisms and haplotypes in the caspase-3, caspase-7, and caspase- 8 genes and risk for endometrial cancer: A population-based, case-control study in a Chinese population. Cancer Epidemiol Biomarkers Prev 18: 2114-2122, 2009.

31. Jang JS, Kim KM, Choi JE, Cha SI, Kim CH, Lee WK, Kam S, Jung TH and Park JY: Identification of polymorphisms in the Caspase-3 gene and their association with lung cancer risk. Mol Carcinog 47: 383-390, 2008.

32. Gosser YQ, Nomanbhoy TK, Aghazadeh B, Manor D, Combs C, Cerione RA and Rosen MK: C-terminal binding domain of Rho GDP-dissociation inhibitor directs N-terminal inhibitory peptide to GTPases. Nature 387: 814-819, 1997.

33. Platko JV, Leonard DA, Adra CN, Shaw RJ, Cerione RA and Lim B: A single residue can modify target-binding affinity and activity of the functional domain of the Rho-subfamily GDP dissociation inhibitors. Proc Natl Acad Sci USA 92: 2974-2978, 1995.

34. Golovanov AP, Chuang TH, DerMardirossian C, Barsukov I, Hawkins D, Badii R, Bokoch GM, Lian LY and Roberts GC: Structure-activity relationships in flexible protein domains: Regulation of rho GTPases by RhoGDI and D4 GDI. J Mol Biol 305: 121-135, 2001

35. Ota T, Maeda M, Okamoto M and Tatsuka M: Positive regulation of Rho GTPase activity by RhoGDIs as a result of their direct interaction with GAPs. BMC Syst Biol 9: 3, 2015. 